\title{
Color difference threshold determination for acrylic denture base resins
}

\author{
Jiabao Ren ${ }^{\mathrm{a}, \mathrm{b}}$, Hong Lin ${ }^{\mathrm{a}, \mathrm{b}}$, Qingmei Huang ${ }^{\mathrm{c}}$, Qifan Liang ${ }^{\mathrm{d}}$ and Gang Zheng,b, \\ ${ }^{a}$ Department of Dental Materials, School of Stomatology, Peking University, Beijing, China \\ ${ }^{b}$ National Engineering Laboratory for Digital and Material Technology of Stomatology, Peking \\ University, Beijing, China \\ ${ }^{c}$ National Laboratory of Color Science \& Engineering, School of Information Science \& Technology, \\ Beijing Institute of Technology, Beijing, China \\ ${ }^{d}$ Department of Actuarial Science, Boston University, Boston, MA, US
}

\begin{abstract}
This study aimed to set evaluation indicators, i.e., perceptibility and acceptability color difference thresholds, of color stability for acrylic denture base resins for a spectrophotometric assessing method, which offered an alternative to the visual method described in ISO 20795-1:2013. A total of 291 disk specimens $50 \pm 1 \mathrm{~mm}$ in diameter and $0.5 \pm 0.1 \mathrm{~mm}$ thick were prepared (ISO 20795-1:2013) and processed through radiation tests in an accelerated aging chamber (ISO 7491:2000) for increasing times of 0 to 42 hours. Color alterations were measured with a spectrophotometer and evaluated using the CIE $\mathrm{L} * \mathrm{a} * \mathrm{~b} *$ colorimetric system. Color differences were calculated through the CIEDE2000 color difference formula. Thirty-two dental professionals without color vision deficiencies completed perceptibility and acceptability assessments under controlled conditions in vitro. An S-curve fitting procedure was used to analyze the 50:50\% perceptibility and acceptability thresholds. Furthermore, perceptibility and acceptability against the differences of the three color attributes, lightness, chroma, and hue, were also investigated. According to the S-curve fitting procedure, the 50:50\% perceptibility threshold was $1.71 \Delta \mathrm{E}_{00}$ $\left(\mathrm{r}^{2}=0.88\right)$ and the $50: 50 \%$ acceptability threshold was $4.00 \Delta \mathrm{E}_{00}\left(\mathrm{r}^{2}=0.89\right)$. Within the limitations of this study, $1.71 / 4.00$ $\Delta \mathrm{E}_{00}$ could be used as perceptibility/acceptability thresholds for acrylic denture base resins.
\end{abstract}

Keywords: Acrylic denture base resin, perceptibility threshold, acceptability threshold, spectrophotometer, CIEDE2000

\section{Introduction}

Evaluations of color stability for denture base resins continue to be a concern in dental materials testing. Currently, the basis for color stability evaluations of acrylic denture base polymers is ISO standard 20795-1:2013 [1], based on the visual assessments of 3 observers, which is subjective and subject to the observers' physical and psychological status and uncontrolled viewing conditions [2-4]. Color measurements performed with accurate color measuring systems can overcome the shortcomings of inconsistent visual methods in shade-matching, bleaching, and quality control $[2,5]$. To provide an objective standard for instrumental assessments of color stability of acrylic denture base

\footnotetext{
* Address for correspondence: Gang Zheng, Department of Dental Materials, School and Hospital of Stomatology, Peking University, No.22 South Avenue Zhongguancun, Haidian District, Beijing, 100081, China. Tel.: +86 010 82195748; Fax: +8601062164691; E-mail: zhengang101@126.com.
} 
resins, color difference indicators, including perceptibility and acceptability thresholds, have been exhaustively studied in dental materials that mimic tooth or skin colors [5-13] and have been recommended by ISO technical report 28642 [14], need to be investigated.

Several instruments, such as colorimeters, spectrophotometers, spectroradiometers, and digital cameras, are widely used in color measurements in dentistry [2, 9, 15]. Their basic structures and measuring principles have been studied and compared previously [2]. Spectrophotometric measurements have been proved to outperform visual assessments in both reliability and reproducibility [16-19], and they are the gold standard of color measuring devices [12]. However, it has been demonstrated that both spectrophotometric and colorimetric devices can suffer edge loss effects, which happen when a device with a small window measuring a transparent material results in light scattering to the edge of the sample without being detected. Edge-loss is affected by the illuminating beam size and direction, observation geometry, thickness of the specimen, and the reflectance of the backing [20].

The perceptibility threshold (PT) and acceptability threshold (AT) have been well studied in dental materials such as tooth-colored resin composites, ceramics, and skin-colored maxillofacial elastomers $[7,8,10,11]$. However, very few studies have focused on the thresholds of instrumental analysis of the color stability of acrylic denture base resins [21]. To date, there are no unanimously agreed upon PT and AT values for dental materials due to discrepancies in methodologies such as differences in the materials tested, different observers, psychophysical methods used, and the color-measuring devices and color difference formulas that were used [7, 22].

This study aimed to set objective indicators for spectrophotometric assessments of the color stability of acrylic denture base resins through a controlled and reproducible lab assessing procedure. Furthermore, dependences of visual perceptibility and acceptability against the three color attributes, lightness, chroma, and hue, were also investigated.

\section{Experimental method}

\subsection{Specimen preparation}

Acrylic denture base powder and liquid (shade \#3, New Century, Shanghai, China) were mixed, condensed into standardized stainless steel molds, covered with a transparent polyester strip on the interface of the material and the mold cover, and pressed in a hydraulic press (Model C, Fred S. Carver, Inc.) to remove excess material. Finally, the specimens were removed after being cured in accordance with the specifications of ISO 20795-1:2013 and the manufacturer's instructions. All specimens were measured with a digital micrometer (Mitutoyo, Japan) and specimens beyond the range of $50 \pm 1 \mathrm{~mm}$ in diameter and $0.5 \pm 0.1 \mathrm{~mm}$ thick were discarded. After a careful examination with the naked eye, disks with flat surfaces and no visible pores were chosen. In total, 291 specimens were selected for testing.

Accelerated aging was used to simulate possible color changes of the acrylic denture base resin during service in the oral cavity; this is a time-saving and reliable method for assessing color stability and has been proved by previous literature [23]. Specimens with aluminum foil covering half of their surface were put through the accelerated aging process in a chamber (Suntest XLS+, Atlas) under radiation conditions recommended by ISO 7491:2000 [24] for a gradient increasing exposure time of 0 to 42 hours to create a color pool large enough from imperceptible to unacceptable. 


\subsection{Color measurement and color difference calculation}

A portable spectrophotometer (SP62, X-rite) was used to measure the reflectance of the specimens and then converted the measurements into CIELAB tristimulus $L^{*}, a^{*}$, and $b^{*}$ values under CIE1964 $10^{\circ}$ observer. The spectrophotometer was calibrated through manufacturer-supplied calibration standards before being used. The measuring system was set to D65 illuminant with measurement geometry of $\mathrm{d} / 8^{\circ}$. Measurements were repeated 5 times on each half of the specimens on a white background (with a reflectance higher than $95 \%$ among $400-700 \mathrm{~nm}$ ), and the mean values of $\mathrm{L}^{*}, \mathrm{a}^{*}$, and $\mathrm{b}^{*}$ were calculated.

The CIEDE2000 color difference formula previously proved to be the best [25] was used to calculate the color change of each specimen between the exposed half and the unexposed half. To achieve better predictability of calculated color differences, it advanced the previous CIE94 formula in four aspects: a scaling factor $\mathrm{a}^{*}$ to improve performance for gray colors, a chroma- hue rotation item $\left(\mathrm{R}_{\mathrm{T}}\right)$ to improve predictability in blue colors, and new weighting functions for lightness and hue $\left(\mathrm{S}_{\mathrm{L}} \&\right.$ $\mathrm{S}_{\mathrm{H}}$ ) to optimize the weightings of $\Delta \mathrm{C}^{\prime}$ and $\Delta \mathrm{H}^{\prime}$ in total $\Delta \mathrm{E}_{00}$ color difference.

It was calculated as follows:

$$
\Delta \mathrm{E}_{00}=\sqrt{\left(\frac{\Delta \mathrm{L}^{\prime}}{\mathrm{k}_{\mathrm{L}} \mathrm{S}_{\mathrm{L}}}\right)^{2}+\left(\frac{\Delta \mathrm{C}^{\prime}}{\mathrm{k}_{\mathrm{C}} \mathrm{S}_{\mathrm{C}}}\right)^{2}+\left(\frac{\Delta \mathrm{H}^{\prime}}{\mathrm{k}_{\mathrm{H}} \mathrm{S}_{\mathrm{H}}}\right)^{2}+\mathrm{R}_{\mathrm{T}}\left(\frac{\Delta \mathrm{C}^{\prime}}{\mathrm{k}_{\mathrm{C}} \mathrm{S}_{\mathrm{C}}}\right)\left(\frac{\Delta \mathrm{H}^{\prime}}{\mathrm{k}_{\mathrm{H}} \mathrm{S}_{\mathrm{H}}}\right)}
$$

\subsection{Visual perceptibility and acceptability assessments}

A total of 32 dental professionals (17 women and 5 men) with an average shade-matching experience of 5 years were screened by a color vision deficiency test using Ishihara charts and then enrolled in the visual assessments portion of this study. All the observers had previously demonstrated a higher discriminability through ROC analysis. Visual perceptibility and acceptability assessments were performed in a dark room in a standard viewing cabinet illuminated with D65 standard artificial daylight (color temperature: 6,500 K). The specimens were positioned on the floor of the cabinet, which was painted a neutral gray, with $0^{\circ}$ illuminating geometry. All observers' heads were fixed at a $45^{\circ}$ angle superior to the horizontal plane at a distance of $30 \mathrm{~cm}$. After 5 minutes' adaptation under the conditions, the observers were instructed to quickly answer the questions in 2 seconds. The first question was "Could you see a color difference between the exposed and unexposed halves?" This question was used to determine the perceptibility threshold. If the answer was yes, then the next question was asked: "Is the color change acceptable?" This was used to determine the acceptability threshold. The percent of positive answers (perceptible answers) in the perceptibility judgments and the percent of negative answers (unacceptable answers) in the acceptability judgments against each specimen were calculated. Both the PT and AT were determined at the 50:50 perceptible/imperceptible or acceptable/unacceptable color difference points. A total of 291 specimens were evaluated by each observer, for a total of 9,312 evaluations made among the 32 observers.

\subsection{S-curve fitting}

The visual perceptible percent against the calculated color difference $\Delta \mathrm{E}_{00}$ were fitted using the scurve with the equation $\mathrm{y}=1 /\left[1+\exp \left(-\mathrm{B}^{*} \mathrm{x}+\mathrm{A}\right)\right]$. The same was applied to the AT threshold. 
Perceptibility and acceptability were also plotted against the three color attributes, which included lightness, chroma, and hue, and then fitted using the s-curve analysis.

\section{Results}

\subsection{Color difference distribution of 291 specimens}

The CIEDE2000 color difference range of all the specimens was 0.23-7.77 with a mean and standard deviation of $3.93 \pm 2.04$. A large color pool from indistinguishable to unacceptable was generated. The distribution of the three attributes of discoloration (lightness, chroma, and hue) differences designated as $\Delta \mathrm{L}^{\prime}, \Delta \mathrm{C}^{\prime}$, and $\Delta \mathrm{H}^{\prime}$ were as shown in Figures 1-3. The lightness mainly decreased when $\Delta \mathrm{E}_{00}$ was less than 4 , while it showed the opposite trend beyond 4 (Figure 1). The chroma sometimes decreased since a relative increase in yellowness-blueness b' cannot make up for a decrease in redness-greenness a' (Figure 2). The hue difference against $\Delta \mathrm{E}_{00}$ showed an increasing trend (Figure 3). The contribution of the changes of these three attributes to the total color difference $\left(\Delta \mathrm{E}_{00}\right)$ of each specimen $\left|\Delta \mathrm{L}^{\prime} / \Delta \mathrm{E}_{00}\right|,\left|\Delta \mathrm{C}^{\prime} / \Delta \mathrm{E}_{00}\right|$, and $\left|\Delta \mathrm{H}^{\prime} / \Delta \mathrm{E}_{00}\right|$ were as shown in Figure 4.

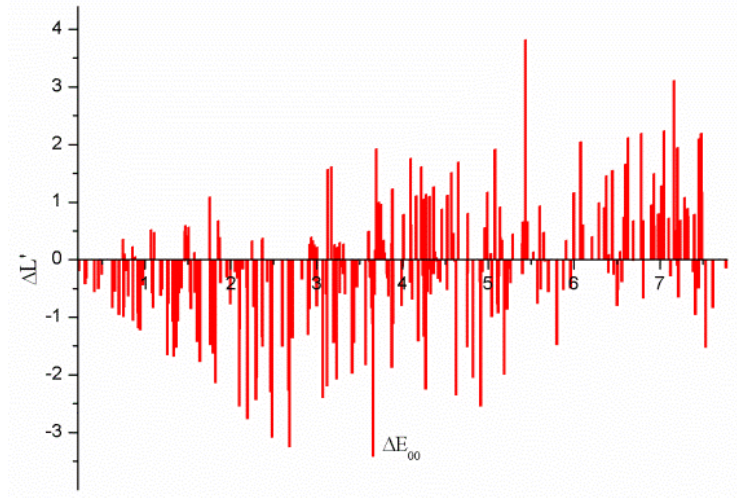

Fig. 1. Distribution of lightness difference in CIEDE2000 color difference of each specimen.

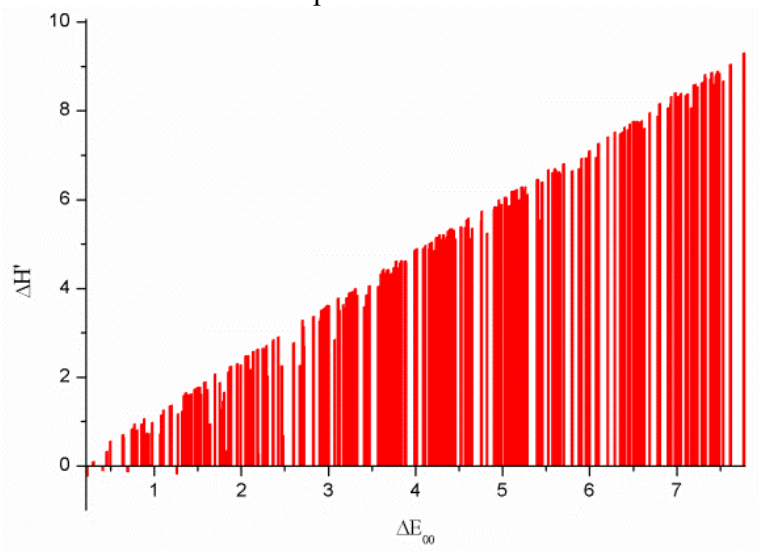

Fig. 3. Distribution of hue difference in CIEDE2000 color difference of each specimen.

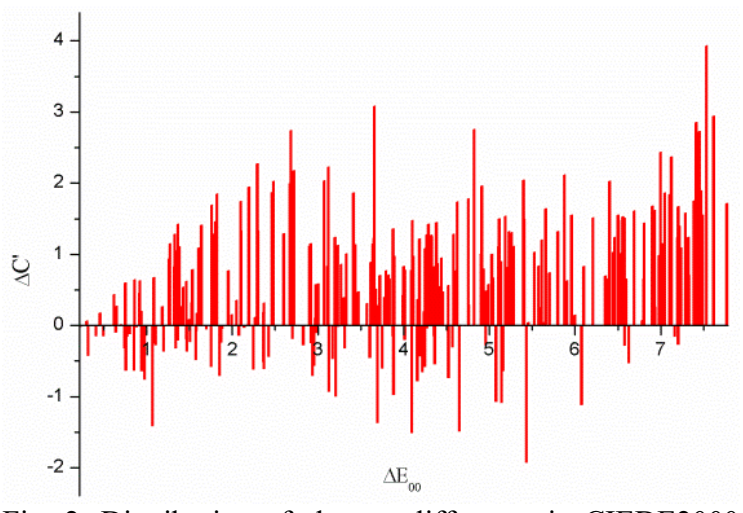

Fig. 2. Distribution of chroma difference in CIEDE2000 color difference of each specimen.

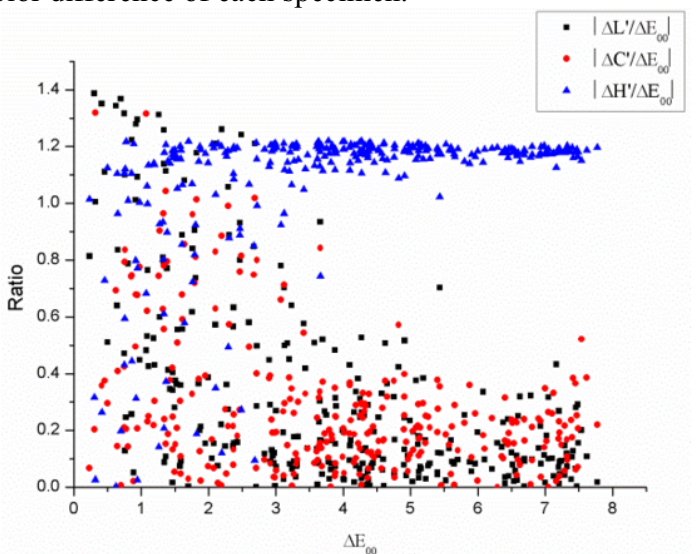

Fig. 4. Contributions of lightness, chroma, and hue differences within each specimen. 


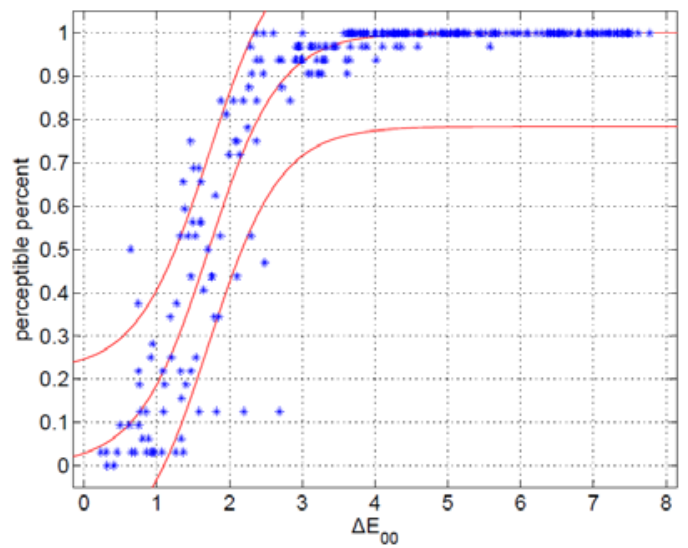

Fig. 5. Perceptible percent versus CIEDE2000 color differences of all specimens.

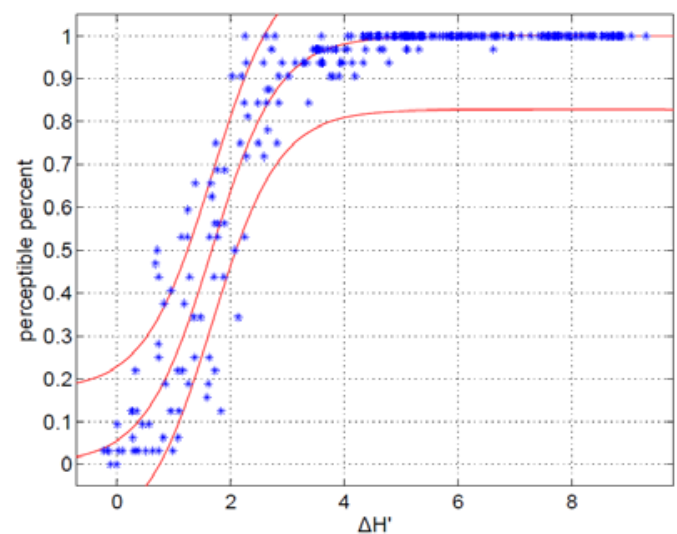

Fig. 7. Perceptible percent versus hue differences of all specimens.

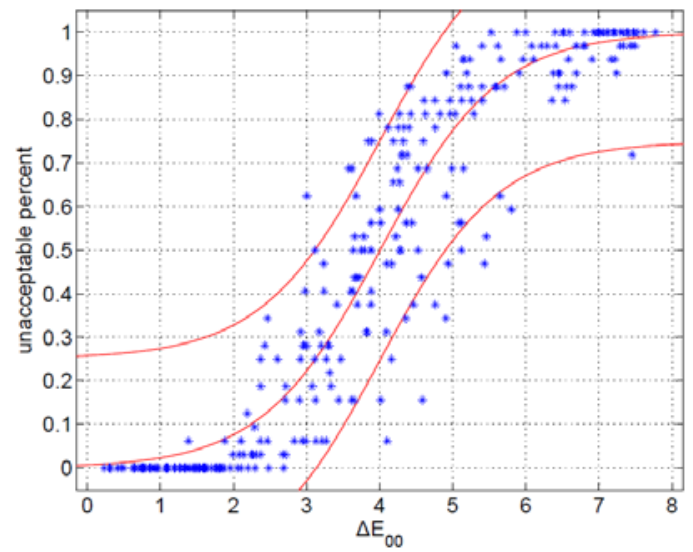

Fig. 6. Unacceptable percent versus CIEDE2000 color differences of all specimens.

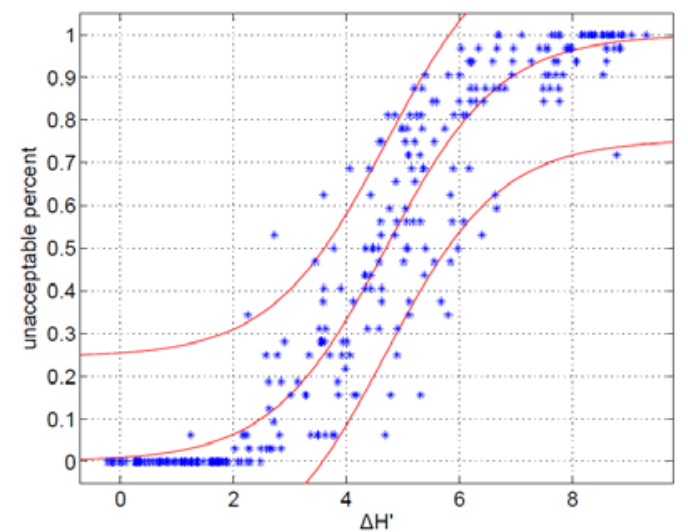

Fig. 8. Unacceptable percent versus hue differences of all specimens.

\subsection{Perceptibility and acceptability threshold determinations}

The percentage of positive answers during the visual perceptibility judgment of all the observers plotted against color difference $\Delta \mathrm{E}_{00}$ was scattered, as shown in Figure 5. An s-curve fitted these scattered points with the coefficients: $\mathrm{A}=3.527, \mathrm{~B}=2.057$, and $\mathrm{r}^{2}=0.88$. The color difference for the 50:50 perceptibility threshold was $1.71 \Delta \mathrm{E}_{00}$ (with $95 \%$ CI 1.36-2.17). Similarly, the percentage of unacceptable answers against $\Delta \mathrm{E}_{00}$ was also scattered, as shown in Figure 6. The coefficients were: $\mathrm{A}=4.978, \mathrm{~B}=1.243$, with $\mathrm{r}^{2}=0.89$. The color difference for the $50: 50$ acceptability threshold was 4.00 $\Delta \mathrm{E}_{00}$ (with $95 \%$ CI 3.27-4.91).

\subsection{PT and AT threshold analyses against lightness, chroma, and hue differences}

The percentages of "perceptible" and "unacceptable" were plotted against $\Delta \mathrm{L}$ ', $\Delta \mathrm{C}$ ', and $\Delta \mathrm{H}$ '. The percentages of "perceptible" and "unacceptable" plotted against $\Delta \mathrm{L}$ ' and $\Delta \mathrm{C}$ ' were scattered irregularly, thus they were not presented in this article. The percentages plotted against $\Delta \mathrm{H}^{\prime}$ ' were as shown in Figures 7 and 8. In the perceptible judgments, the fitted s-curve had the coefficients of 
$\mathrm{A}=2.835$ and $\mathrm{B}=1.702$, with $\mathrm{r}^{2}=0.93$. The hue difference for the $50: 50$ perceptibility was $1.67 \Delta \mathrm{E}_{00}$ (with $95 \%$ CI 1.37-2.02). Similarly, the coefficients fitted in the acceptable judgments were $A=4.669$ and $\mathrm{B}=0.9944$, with $\mathrm{r}^{2}=0.89$. The hue difference for the $50: 50$ acceptability threshold was $4.70 \Delta \mathrm{E}_{00}$ (with 95\% CI 3.83-5.75).

\section{Discussion}

\subsection{Color changes}

Acrylic denture bases suffer from various conditions during clinical services in the oral cavity, such as saliva washout, moisture, foods and liquids, smoke, and masticatory stress [26]. A controlled clinical study could reasonably ascertain the color stability over time. However, it would be time consuming; therefore, the accelerated artificial aging method for testing color stability described in ISO 7491:2000 was used. The discoloration during accelerated aging may result from internal reactions such as amine accelerator discoloration upon oxidation and residual monomer content in the curing system $[23,27]$. There is no consensus on the unacceptable value of color deformation. Douglas reported an unacceptable value of 5.5 CIELAB units $\left(\Delta \mathrm{E}^{*}\right)$ [6]. Johnston and Kao consider $3.7 \Delta \mathrm{E}^{*}$ as a match and $6.8 \Delta \mathrm{E}^{*}$ as a mismatch between teeth compared in the oral cavity [28]. These studies only had a limited number of specimens and narrow color difference ranges. To make up for the limitations of previous studies, a large and more evenly distributed color pool was created for this study.

\subsection{Color difference formula}

An appropriate color difference formula would achieve a better fit between calculated and visually observed color differences. The CIEDE2000 color difference formula is the color difference formula recently recommended by CIE. The study by Luo, et al. [29], which tested the CIELAB and advanced CIELAB based equations using four color discrimination datasets (mean $2.6 \Delta \mathrm{E}^{*}$ ) and found CIEDE2000 performed the best. It was also found by the authors that CIEDE2000 color difference formula possesses higher discriminability especially when doing perceptible judgments by ROC analysis. The results of the present study are in agreement with several previous studies in dentistry [7, 11]. These studies found that CIEDE2000 provided a higher degree of fit when evaluating color differences of dental materials $[7,11]$.

\subsection{Perceptibility and acceptability thresholds}

The 50:50 perceptibility/acceptability threshold obtained in this study was $1.71 / 4.00 \Delta \mathrm{E}_{00}$, which was higher than previously reported values. Ghinea, et al. studied color difference thresholds of ceramic discs within the color range of central and lateral incisor and canine teeth and reported corresponding PT/AT values of 1.30/2.25 $\Delta \mathrm{E}_{00}$ by an S-fitting procedure [7]. Paravina, et al. studied the PT/AT of elastomers under a different D50 artificial light (color temperature: 5,000 K) and suggested values of $0.7 / 2.1 \Delta \mathrm{E}_{00}$ and $1.2 / 3.1 \Delta \mathrm{E}_{00}$, respectively, for light and dark shade elastomers mimicking the skin color of Caucasians and African-Americans [8]. This may be a result of the discrepancies in visual color tolerance of different regions in the CIELab color space [30]. Dental professionals tend to have greater visual tolerance for pink-colored denture base materials than tooth- 
colored materials. Although these studies were all carried out in vitro, distinctions still exist in the methodologies used such as the color measuring systems, composition of observers, and differences in illuminating; viewing geometries and conditions designated as "parametric effects" may also play a role [31].

\subsection{Perceptibility versus acceptability}

The PT obtained in this study was evidently smaller than the AT, which is contrary to several previous studies. Decades ago, Kuehni, et al. carried out perceptibility and acceptability judgments assessing small color differences based on six color centers and found equivalent $\triangle \mathrm{E}^{*} \mathrm{~s}$ (CIELAB color difference values) for the perceptibility and acceptability thresholds [32]. However, Kuehni's experiment was not based on the color of the dental materials. Recently, Lindsey, et al., assessed color difference thresholds through computer-generated tooth color stimulus and concluded that there were no significant differences between the PT and AT [22]. Discrepancies exist between literatures and this study in terms of both psychophysical visual assessment design and materials used. A more critical asking strategy was undertaken when assessing the AT in Lindsey's design. However, in the present study, the observers performed perceptibility and acceptability judgments in a sequential procedure, i.e., they were asked to do acceptability judgments only when they answered "yes" in the perceptibility judgments. This might have caused the discrepancies in the observed results, and was consequently confirmed in a previous study [7].

This study attempted to determine perceptibility and acceptability thresholds against lightness, chroma, and hue differences. However, the control of a single variable in the color attributes, lightness, chroma, or hue, is very hard to realize during specimen age-accelerating processes. Thus, the perceptibility and acceptability judgments were made based on the combination effects of the color attribute changes (lightness, chroma, and hue changes) within each specimen. That is to say, the 1.67/4.70 $\Delta \mathrm{E}_{00} \mathrm{PT} / \mathrm{AT}$ values against hue were not simply hue-dependent visual perception thresholds, but thresholds derived from hue, chroma and lightness changes. Furthermore, it could be concluded that the color difference thresholds determination in this study were dominated by hue alteration. Though sizable literatures have explored the perceptibility and/or acceptability of dental materials, they seldom mention the separate contribution of lightness, chroma, and hue changes to the total calculated color difference. This may also be a source of distinction in the PT/AT values compared to other studies.

\subsection{Limitations and future directions}

Color measurements in this study suffered from edge loss effects $[9,20]$. To minimize the amount of edge-loss effects, the specimens were made of a somewhat large diameter $(50 \mathrm{~mm})$ of flat surface and color was measured on a highly-reflective white backing. This study indicated that PT and AT thresholds correlated with the relative contribution of lightness, chroma, and hue differences $\left(\Delta \mathrm{L}^{\prime}, \Delta \mathrm{C}^{\prime}\right.$, $\left.\Delta \mathrm{H}^{\prime}\right)$ to the total color difference of the tested samples. Thus, whether the relative contribution of $\Delta \mathrm{L}^{\prime}$, $\Delta \mathrm{C}^{\prime}$, and $\Delta \mathrm{H}^{\prime}$ in the present study would be appropriate to represent all acrylic denture base resins of different brands and shades needs to be investigated further.

\section{Conclusion}


Within the limitations of this study, it was found that the perceptibility/acceptability thresholds $1.71 / 4.00 \Delta \mathrm{E}_{00}$ can be used as an indicator for the color stability of acrylic denture base resins and that the relative contribution of the three color attributes to the total calculated $\Delta \mathrm{E}_{00}$ played a role in the color difference determination of the acrylic denture base resins.

\section{References}

[1] Dentistry -- Base polymers -- Part 1: Denture base polymers (ISO 20795-1:2013), available at: http://www.iso.org/iso/home.htm, last accessed Feb. $25^{\text {th }}, 2015$.

[2] H. Chen, et al., A systematic review of visual and instrumental measurements for tooth shade matching, Quintessence International 43 (2012), 649-659.

[3] B.S. Kim, et al., Shade comparative analysis of natural tooth measured by visual and spectrophotometric methods, The Journal of Korean Academy of Prosthodontics 46 (2008), 443-454.

[4] S.A. Bahannan, Shade matching quality among dental students using visual and instrumental methods, Journal of Dentistry 42 (2014), 48-52.

[5] M.D.M. Perez, et al., Dental ceramics: A CIEDE2000 acceptability thresholds for lightness, chroma and hue differences, Journal of Dentistry 39 (2011), e37-e44.

[6] R.D. Douglas, et al., Intraoral determination of the tolerance of dentists for perceptibility and acceptability of shade mismatch, Journal of Prosthetic Dentistry 97 (2007), 200-208.

[7] R. Ghinea, et al., Color difference thresholds in dental ceramics, Journal of Dentistry 38 (2010), e57-e64.

[8] R.D. Paravina, et al., Color difference thresholds of maxillofacial skin replications, Journal of Prosthodontics-Implant Esthetic and Reconstructive Dentistry 18 (2009), 618-625.

[9] W.M. Johnston, Color measurement in dentistry, Journal of Dentistry 37 (2009), e2-e6.

[10] N. Alghazali, et al., Assessment of perceptibility and acceptability of color difference of denture teeth, Journal of Dentistry 40 (2012), e10-e17.

[11] A.G. Wee, et al., Use of a porcelain color discrimination test to evaluate color difference formulas, Journal of Prosthetic Dentistry 98 (2007), 101-109.

[12] G. Khashayar, et al., Perceptibility and acceptability thresholds for colour differences in dentistry, Journal of Dentistry 42 (2014), 637-644.

[13] S. Ishikawa-Nagai, et al., Clinical evaluation of perceptibility of color differences between natural teeth and all-ceramic crowns, Journal of Dentistry 37 (2009), e57-e63.

[14]Dentistry -- Guidance on colour measurement (ISO/TR 28642:2011). http://www.iso.org/iso/home.htm, last accessed Feb. $25^{\text {th }}, 2015$.

[15] A. Dozic, et al., Performance of five commercially available tooth color-measuring devices, Journal of Prosthodontics 16 (2007), 93-100.

[16] P. Gehrke, et al., Comparison of in vivo visual, spectrophotometric and colorimetric shade determination of teeth and implant-supported crowns, International Journal of Computerized Dentistry 12 (2009), 247-263.

[17] S. AlSaleh, et al., Evaluation of self-shade matching ability of dental students using visual and instrumental means, Journal of Dentistry 40 (2012), e82-e87.

[18] S. Paul, et al., Visual and spectrophotometric shade analysis of human teeth, Journal of Dental Research 81 (2002), 578582.

[19] C. Odaira, et al., Clinical evaluation of a dental color analysis system: The crystaleye spectrophotometer, Journal of Prosthodontic Research 55 (2011), 199-205.

[20] W.M. Johnston, et al., Analysis of edge-losses in reflectance measurements of pigmented maxillofacial elastomer, Journal of Dental Research 75 (1996), 752-760.

[21] L.L. Lou, et al., Comparative study on spectrophotometric and visual methods for color stability of denture base resin, Chinese Journal of Stomatology 46 (2011), 241-244.

[22] D.T. Lindsey, et al., Perceptibility and acceptability of CIELAB color differences in computer-simulated teeth, Journal of Dentistry 35 (2007), 593-599.

[23]X. Wang, et al., Color stability of heat-activated and chemically activated fluid resin acrylics, Journal of Prosthodontics 5 (1996), 266-269.

[24] Dental materials -- Determination of colour stability (ISO 7491:2000). http://www.iso.org/iso/home.htm, last accessed Feb. $25^{\text {th }}, 2015$. 
[25] M.D. Perez, et al., Study of the variation between CIELAB Delta E* and CIEDE2000 color-differences of resin composites, Dental Materials Journal 26 (2007), 21-28.

[26] M.A. Arocha, et al., Colour stainability of indirect CAD-CAM processed composites vs. conventionally laboratory processed composites after immersion in staining solutions, Journal of Dentistry 42 (2014), 831-838.

[27] M.R. Bonatti, et al., The effect of polymerization cycles on color stability of microwave-processed denture base resin, Journal of Prosthodontics-Implant Esthetic and Reconstructive Dentistry 18 (2009), 432-437.

[28] W.M. Johnston and E.C. Kao, Assessment of appearance match by visual observation and clinical colorimetry, Journal of Dental Research 68 (1989), 819-822.

[29] M.R. Luo, et al., The development of the CIE 2000 colour-difference formula: CIEDE2000, Color Research and Application 26 (2001), 340-350.

[30] M. Melgosa, et al., Suprathreshold color-difference ellipsoids for surface colors, Color Research and Application 22 (1997), 148-155.

[31] S.S. Guan and M.R. Luo, Investigation of parametric effects using small colour differences, Color Research and Application 24 (1999), 331-343.

[32] R.G. Kuehni, et al., An experiment in visual scaling of small color differences, Color Research and Application 4 (1979), 83-91. 